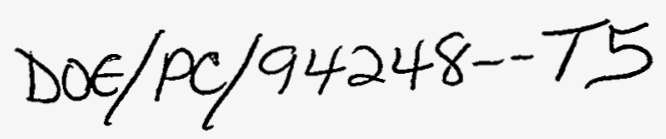

\title{
VELOCITY AND CONCENTRATION STUDIES OF FLOWING SUSPENSIONS BY NUCLEAR MAGNETIC RESONANCE IMAGING
}

(ID: DE-FG22-94PC94248)

Technical Progress Report

to Pittsburgh Energy Technology Center

for the quarter ending 12/31/95

\section{Task 1: Particle selection}

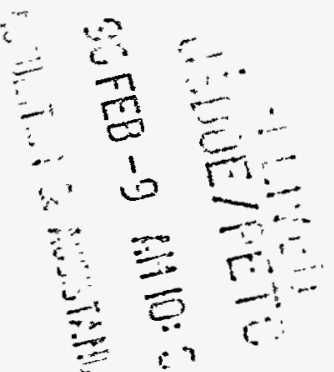

We have recently examined poly(dimethylsiloxane) as a possible liquid to use with water-solfible pharmaceutical pills of the sort we have used to study granular flows. It is available in many viscosities from less than 1 centistokes to 100,000 centistokes or more as viscosity standards. Its specific gravity ranges from 0.93 to 0.975 for viscosities between 10 and 100,000 centistokes although it drops steeply with decreasing viscosity; for example, it is 0.76 for viscosity of 0.65 .

Poly(dimethylsiloxane) does have a weak silicon-29 signal (natural abundance $4.7 \%$, resonance frequency in our magnet $15.96 \mathrm{Mhz}$, and sensitivity $3.7 \times 10^{-4}$ compared to equal number of protons) and we can study a stationary vial of it but it will be much more difficult to measure a flowing sample of it. In fact, this seems so impractical that we are looking for other contrast mechanisms first. The most promising contrast will be the spin-lattice relaxation time $T_{1}$ and we will pursue this avenue in the next few months.

\section{Task 5: Flows in complex geometries}

Shear-induced migration of suspended particles can cause large particle concentration gradients in what initially was a well-mixed suspension (the "demixing" of the suspension). Since the effective viscosity of a suspension depends strongly on particle concentration, this demixing can dominate the rheology of the system, and complicate quality assurance issues when a well dispersed system is desirable. Direct measurements of both velocity and particle distribution in concentrated suspensions are made difficult by the large number of particle-fluid interfaces (which scatter light, sound waves, and particle beams). We, at Lovelace, have pioneered the use of nuclear magnetic resonance (NMR) imaging to make noninvasive, accurate, and reproducible measurements of particle and velocity distributions in highly filled suspensions. Despite this increase in the quantity of flow data available, the behavior of suspensions in complex flows remains largely unknown.

We used NMR imaging to measure the concentration and velocity profiles of two suspensions flowing through a straight pipe into a sudden 4:1, axisymmetric contraction, and out of a sudden 4:1 expansion. Both suspensions are $50 \%$ by volume of roughly monodisperse, spherical particles ( 100 or $675 \mu \mathrm{m}$ in diameter) neutrally buoyant in a viscous Newtonian liquid. In this "creeping" flow, inertial, Brownian, and surface forces are negligibly small. We expected and found that shear induced migration and the attendant non-uniformities of viscosity would result in a flow field that is significantly modified from that of a single-phase Newtonian liquid.

The extruder, shown schematically in Figure 1, consists of three $38 \mathrm{~cm}$ long sections joined endto end. Flow proceeds from an upstream reservoir pipe (Region 1) through a smaller diameter pipe (Region 2) into another larger catch pipe (Region 3). The larger-diameter pipes are $5.08 \mathrm{~cm} \mathrm{ID}$ polymethyl methacrylate (PMMA) tubing with $0.635-\mathrm{cm}$-thick walls. The smaller pipe is made from 6.35-cm-OD PMMA rod with a 1.27-cm-diameter hole bored through the center.

As shown in the figure, the contraction and expansion are abrupt. Two identical pistons, sealed with O-rings, slide in the larger pipes. A long push rod connects a motor-driven screw located across the room (to avoid the magnet). Initially, the reservoir tubing and smaller pipe are filled with suspension, 
and the free piston on the expansion end of the pipe is near, but not necessarily adjoining, the expansion joint. The entire test section is placed coaxially in the magnet and braced at the end opposite the motor. The screw pushes the piston at a steady rate in the reservoir tubing, forcing the suspension to flow through it and into the contraction, through the smaller tube, and into the expansion. The plunger moves at a constant velocity of $0.0625 \mathrm{~cm} / \mathrm{s}$, resulting in a mean velocity of $1.0 \mathrm{~cm} / \mathrm{s}$ in the smaller pipe.

The suspensions are $50 \%$, by volume, of PMMA spherical particles suspended in a viscous liquid with the same density as the particles $\left(1.18 \mathrm{~g} / \mathrm{cm}^{3}\right)$. Two sets of particles were used, both with fairly broad, but unimodal, distributions of diameters. One, Diakon MG102, has a mean diameter of $675 \mu \mathrm{m}$ while the other, Lucite $4 \mathrm{~F}$, has a mean diameter of approximately $100 \mu \mathrm{m}$. The suspending liquid is a solution of practical grade 1,1,2,2 tetrabromoethane (14.07\% by weight); UCON oil (H-90,000), a polyalkylene glycol (35.66\% by weight); and Triton X-100, an alkylaryl polyether alcohol $(50.27 \%$ by weight). This liquid exhibits Newtonian rheology with a viscosity of $4.95 \mathrm{~Pa}$ s at $23.15^{\circ} \mathrm{C}$.

The basic NMRI techniques of velocity measurement used in this work were similar to those used earlier in our granular flow experiments [M. Nakagawa, S. A. Altobelli, A. Caprihan, E. Fukushima, and E.-K. Jeong, "Non-invasive Measurements of Granular Flows by Magnetic Resonance Imaging," Experiments in Fluids, 1993, 16:54-60] but, hardware improvements allowed us to create images in shorter times (static images were performed in 4 minutes and each velocity component was measured in two minutes). Image slice thickness was $0.5 \mathrm{~cm}$, and in-plane spatial resolution was approximately 0.2 $\mathrm{mm}$ yielding a voxel size of $0.5 \times 0.5 \times 5 \mathrm{~mm}^{3}$. As with previous work, the time-averaged location and flow of the liquid phase of suspensions is measured with a NALORAC Quest 4400 imager interfaced with a $1.9 \mathrm{~T}, 31 \mathrm{~cm}$ horizontal bore magnet

We imaged various slices of the suspension-filled device within the magnet, including three cross-sections along the length of Region 2, single cross sections in Regions 1 and 3, and slices containing the axis of symmetry in Regions 1 and 3, (Region numbers refer to Figure 1). Velocity images were obtained with conventional pulse gradient spin-echo imaging sequences in which a single, selectable, velocity component is encoded in the phase of the image; static reference images were used to remove extraneous contributions to the phase. The velocity images represent a time average whereas the concentration images show the conditions obtained after moving the piston a known distance. Static images confirmed that the suspension in the upstream reservoir was initially well-mixed.

Figure 3 shows static concentration images of the suspension of larger particles $(675 \mathrm{~mm})$ flowing through the extruder. There are two slice orientations: circular cross-sections (a, $g$ ) and horizontal slices containing the center line $(b-f, h-j)$. Because our NMR imager has an effective region for imaging that is a $7-8 \mathrm{~cm}$ diameter sphere, the extruder extends beyond this region. Thus, the horizontal slices have image intensity that drops off on both sides of each image that are artifacts of the magnet homogeneity, coil dimension, filter, etc.

For these large particles, we find that the contraction affects the flow upstream very little while the expansion leads to an interesting concentration and flow pattern. Images a-f were taken in Region I, the contraction end of the model. Images $a$ and $b$ show that the suspension is initially well mixed and images $c$ and d, obtained after the piston moved 2 and 4 large diameters, respectively, show that the distribution of solids remains virtually unchanged. Even images e and $f$, taken after 6 and 6.25 diameters of piston travel, do not show any concentration inhomogeneity associated with the contraction.

Much to our surprise, a sharp high fluid-fraction spike (light colored streaks, corresponding to fluid fraction of 0.55-0.6) appeared along the model centerline when the piston face approached the field of view, i.e., to within a diameter of the contraction (note: the piston can be seen in image f). We believe that this feature is caused by the interaction of the suspension with the piston face and has nothing to do with the presence of the contraction.

Figure 4 shows profiles of fluid fractions at normalized locations $\mathrm{Z} / \mathrm{L}$ of $10 \%, 50 \%$, and $95 \%$ along the small tube (Region 2 of Fig. 1), where $\mathrm{L}=38 \mathrm{~cm}$, for these $675 \mu \mathrm{m}$ spheres. The particles 
migrate toward the center, as expected, even starting from an opposite distribution caused by the previously mentioned spike of high fluid fraction in the center.

The expansion region, Region 3, shows a more dramatic effect of particle migration than Region 1. Figure $3(\mathrm{~g})$, a concentration cross-section $0.5 \mathrm{~cm}$ downstream of the expansion, shows a light colored ring of high fluid content, surrounding a core having a higher solid fraction. As the suspension travels from Region 2 into the larger pipe of Region 3, the high liquid-rich regions along the wall of the small pipe expand outward towards the wall of the large pipe, as shown in Fig. 3(h-j). In Fig. 3(i), the high fluid fraction lines may hint of the existence of recirculation zones immediately downstream of the expansion. After the plunger has traveled 6 large diameters $(30 \mathrm{~cm})$ the particles in the "corners" appear to have been largely swept away, as shown in Figure 3(j), leaving large, somewhat stagnant, regions with $10-15 \%$ higher liquid content.

When a suspension of $50 \%$ by volume of $100 \mu \mathrm{m}$-diameter particles was pushed through the contraction and expansion, the concentration inhomogeneities created by the migration of particles were much less than for the suspension of $675 \mu \mathrm{m}$ particles described above. Figure 5 shows static images of the $100 \mu \mathrm{m}$ suspension after a 6 large section diameter push. Both effects seen with the larger particles, namely, the liquid-rich spike in front of the piston face in Region 1 and the divergence of the fluid-rich layer at the expansion, are discernible albeit faintly. The top image shows the light-colored feature that has formed in front of the piston face while the lower image shows streaks of enhanced fluid fraction emanating from the expansion at a much sharper angle than they did for the $675 \mu \mathrm{m}$ suspension.

Measurements halfway along the small tube of Region 2 showed a blunt velocity profile but no detectable particle migration, except for a thin fluid-rich layer along the wall, as shown in Figure 6 . The suspension, exhibits a blunt profile that can be represented by

$$
\mathrm{V}_{\mathrm{r}}(\mathrm{r})=\overline{\mathrm{V}}\left(1+\frac{2}{2.8}\right)\left(1-\left(\frac{\mathrm{r}}{\mathrm{R}_{\mathrm{s}}}\right)^{2.8}\right) \text {, }
$$

where $R_{s}$ is the radius of the small tube. All of the velocity profiles observed in the small tube section were similar.

Orthogonal velocity components $V_{z}$ along the axis of the model and $V_{x}$ transverse to it in the horizontal plane were measured using the horizontal slice imaging sequence. The top images of Fig. 7 show the longitudinal velocity component $V_{z}$ scaled between 0 and $2 \mathrm{~cm} / \mathrm{s}$. The suspension of $100 \mu \mathrm{m}$ spheres are shown on the left, and the $675 \mu \mathrm{m}$ suspension are shown on the right. The lower two images of Fig. 7 show the transverse velocity component $V_{x}$ whose absolute value is the radial velocity component. These velocity measurements confirm the much steeper angle of divergence of the expansion flow in the suspension of smaller spheres than in the suspension of larger spheres as it was observed in the concentration images as mentioned earlier.

When the driven piston face entered the imaging region, a filamentous fluid-enriched feature was always observed along the model axis, starting from the piston face. This effect was much greater for the suspension of larger particles and is a spectacular, straight and thin, fluid-rich spike. To our knowledge, this effect has never been seen before. We have now confirmed in a separate experiment that the spike forms in front of the piston and travels with it, without regard for any downstream contraction.

Flow into the abrupt contraction by itself did not produce observable particle migration, but flow in the small tube eventually resulted in migration of the large particles toward the axis of the model and concomitant migration of the fluid toward the wall: Migration in the small tube is noticeable compared to the large pipe of Region 1 because the shear rate in the small tube is much higher than in the large tube. Velocity profiles were blunt, even for the smaller particles, compared to fully developed Newtonian fluid profiles, and did not appear to change along the length of the small tube. These observations are at least qualitatively in agreement with predictions based on shear induced particle migration as described by Leighton and Acrivos [Leighton, D. and Acrivos, A., The Shear-Induced Migration of Particles in Concentrated Suspensions. J. Fluid Mech. 1987, 275, 155-199.] 
The inhomogeneities created in the small tube of Region 2 were convected into the larger diameter expansion in Region 3. Static images of fluid fraction and two-dimensional velocity measurements indicate that the flow field in the expansion region depends more strongly on the particle size than does the flow in the other sections of the model. In the small-sphere suspension, the flow diverges more sharply at the expansion than the flow in the larger sphere case, resembling a pure Newtonian fluid. Our two suspensions were made to have the same bulk effective properties but they had vastly different size particles. We conclude that interparticle interactions affect multiphase flows and this effect is made large or small depending on the particle size. Thus, we cannot predict suspension behavior based only on bulk, or "effective", properties.

In conclusion, we can induce complex spatial variations in particle concentration by flowing a suspension through sudden contractions and expansions. The practical ramifications are that it may be difficult to maintain a well-mixed suspension while filling a vessel from an inlet tube having a crosssection smaller than the vessel. The demixing effects are much larger in suspensions of coarse particles than in suspensions of finer particles.

\section{Figure captions}

Figure 1. The extruder assembly is constructed from three $38 \mathrm{~cm}$ long sections press fit together. The inner diameters of the sections were in the ratio $4: 1: 4$. The suspension was loaded into the extruder between two moveable pistons. The entire assembly was inserted into the horizontal bore magnet so that the region of interest was centered in the NMR sensitive region.

Figure 2. Static NMR images of the contraction (a-f) and the expansion (h-j) regions for a suspension that is $50 \%$ by volume of $675-\mu \mathrm{m}$-diameter particles. A and $\mathrm{g}$ are transverse images $0.5 \mathrm{~cm}$ and $1.5 \mathrm{~cm}$ away from the contraction and the expansion, respectively. The remainder are horizontal slides containing the centerline. Images $a$ and $b$ show the initial condition. Images $c, d, e$, and $f$ show the fluid fraction after the piston has moved $2,4,6$, and 6.25 large section diameters, respectively. Images $h, i$, and $\mathrm{j}$ show the expansion region after 2,4 , and 6 diameters of piston travel.

Figure 3. Fluid fraction as a function of the radial coordinate for three stations along the small tube for the suspension of $675-\mu \mathrm{m}$ spheres. $\mathrm{Z}$ is the distance along the small tube and $\mathrm{L}$ is the length of the small tube. At the upstream station the fluid rich region along the axis is seen. In the central and downstream profiles solids progressively accumulated near $r=0$.

Figure 4. Images of liquid fraction in the contraction region (top) and the expansion region (bottom) after 6 diameters of piston travel in the $100 \mathrm{~mm}$ suspension show small areas of slightly enhanced fluid fraction at the driven piston face (top) and at the expansion. The nominal concentration of solids in this suspension is $50 \%$ by volume.

Figure 5. Fluid fraction (asterisks) as a function of radius at the central site in the small tube for the 100 $\mu \mathrm{m}$ suspension is nearly constant. The velocity profile (pluses) fits a power law profile (solid curve).

Figure 6. Images of the axial (top) and transverse (bottom) velocity components for the $100 \mu \mathrm{m}$ (left) and $675 \mu \mathrm{m}$ (right) suspensions are shown. Dark represents fast axial velocity while light represents slow axial velocity. For the transverse velocity, dark represents velocities towards the top of the image and light towards the bottom. Velocity units are in $\mathrm{cm} / \mathrm{s}$. 
Miscellaneous:

L. Z. Wang, A. Caprihan, and E. Fukushima, "The narrow pulse criterion for pulsed gradient spin echo diffusion measurements," J. Magn. Reson. A, 1995; 117: 209-219; is a recent publication of work performed with partial support from this grant.

6 February 1996

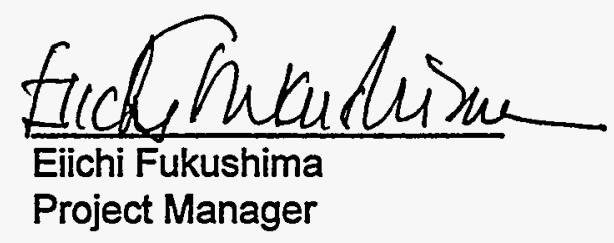

\section{DISCLAIMER}

This report was prepared as an account of work sponsored by an agency of the United States Government. Neither the United States Government nor any agency thereof, nor any of their employees, makes any warranty, express or implied, or assumes any legal liability or responsibility for the accuracy, completeness, or usefulness of any information, apparatus, product, or process disclosed, or represents that its use would not infringe privately owned rights. Reference herein to any specific commercial product, process, or service by trade name, trademark, manufacturer, or otherwise does not necessarily constitute or imply its endorsement, recommendation, or favoring by the United States Government or any agency thereof. The views and opinions of authors expressed herein do not necessarily state or reflect those of the United States Government or any agency thereof. 


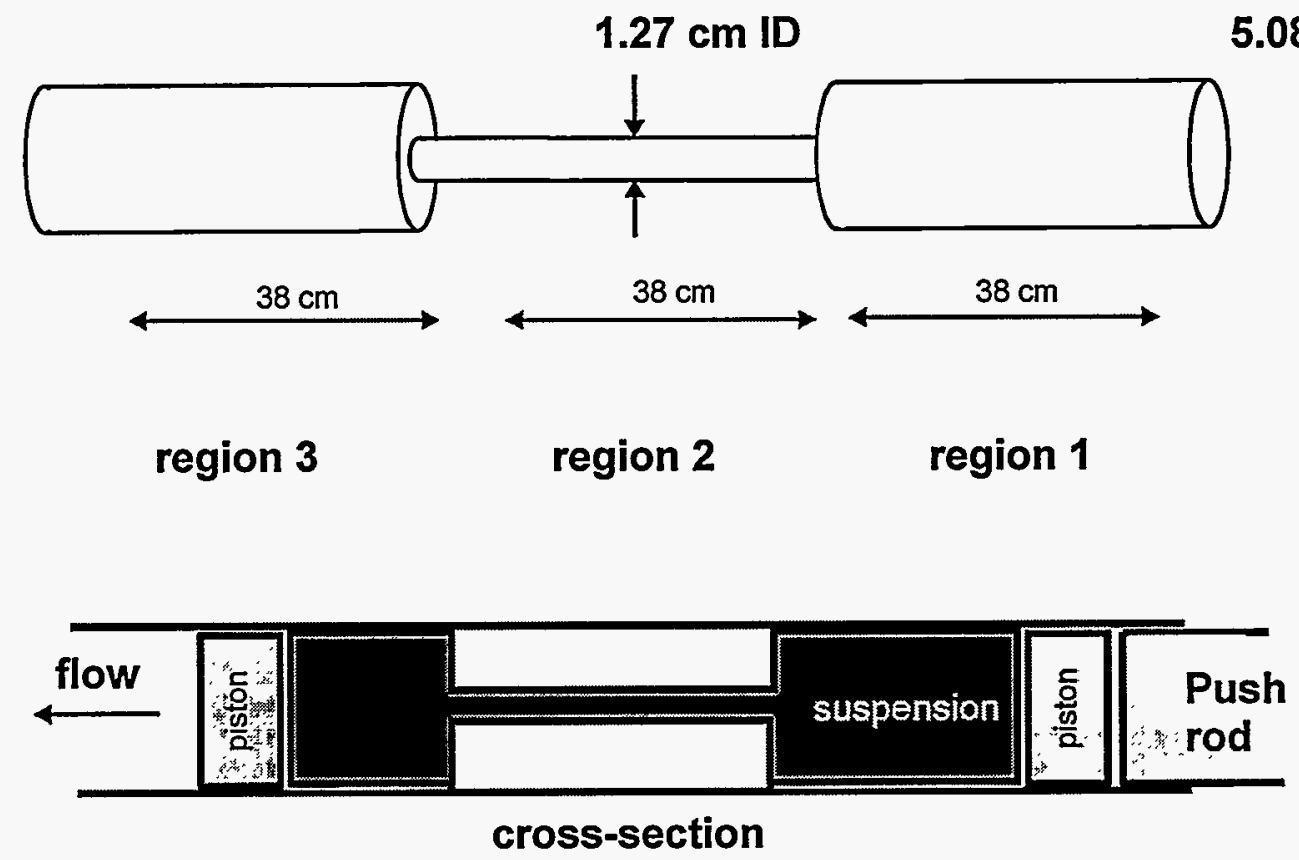

Figure 1 


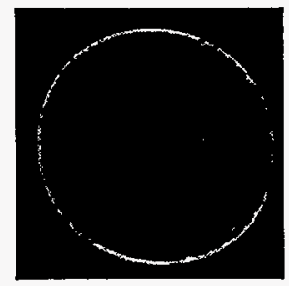

a
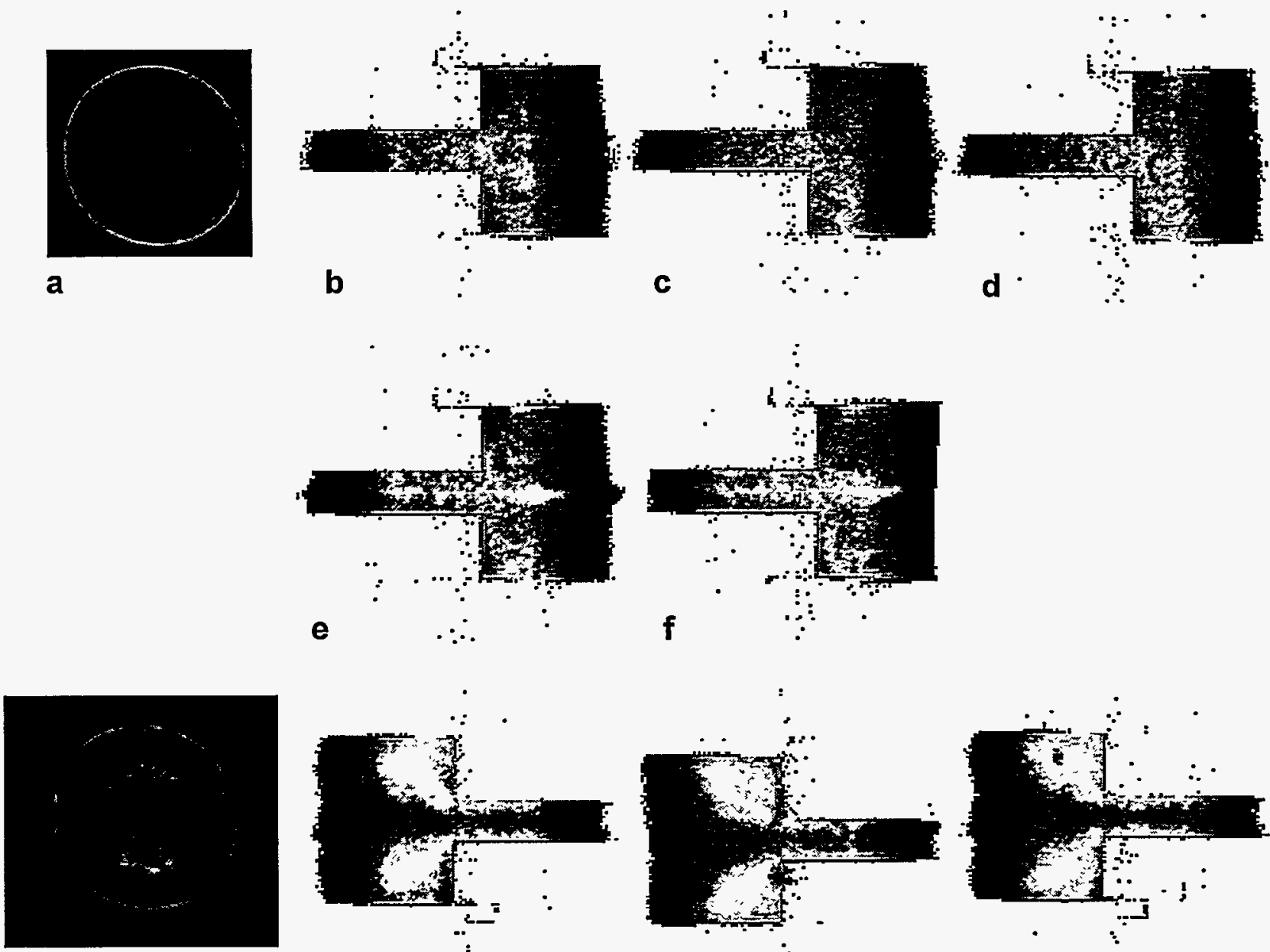

g

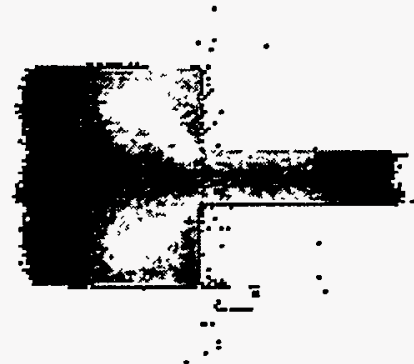

h

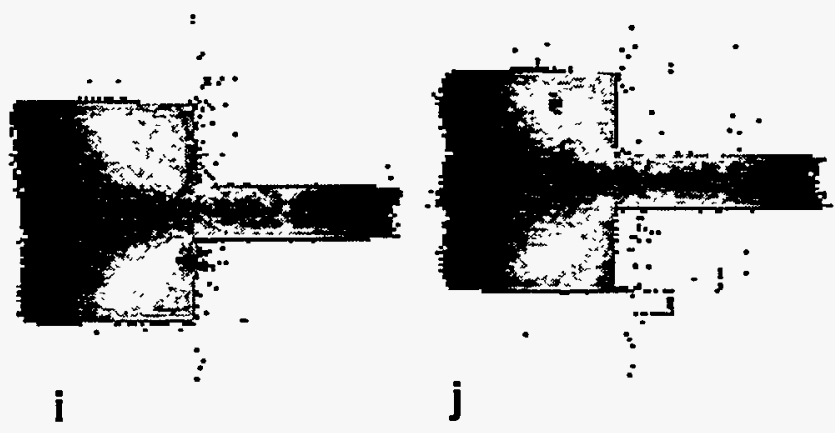

Figure 2 


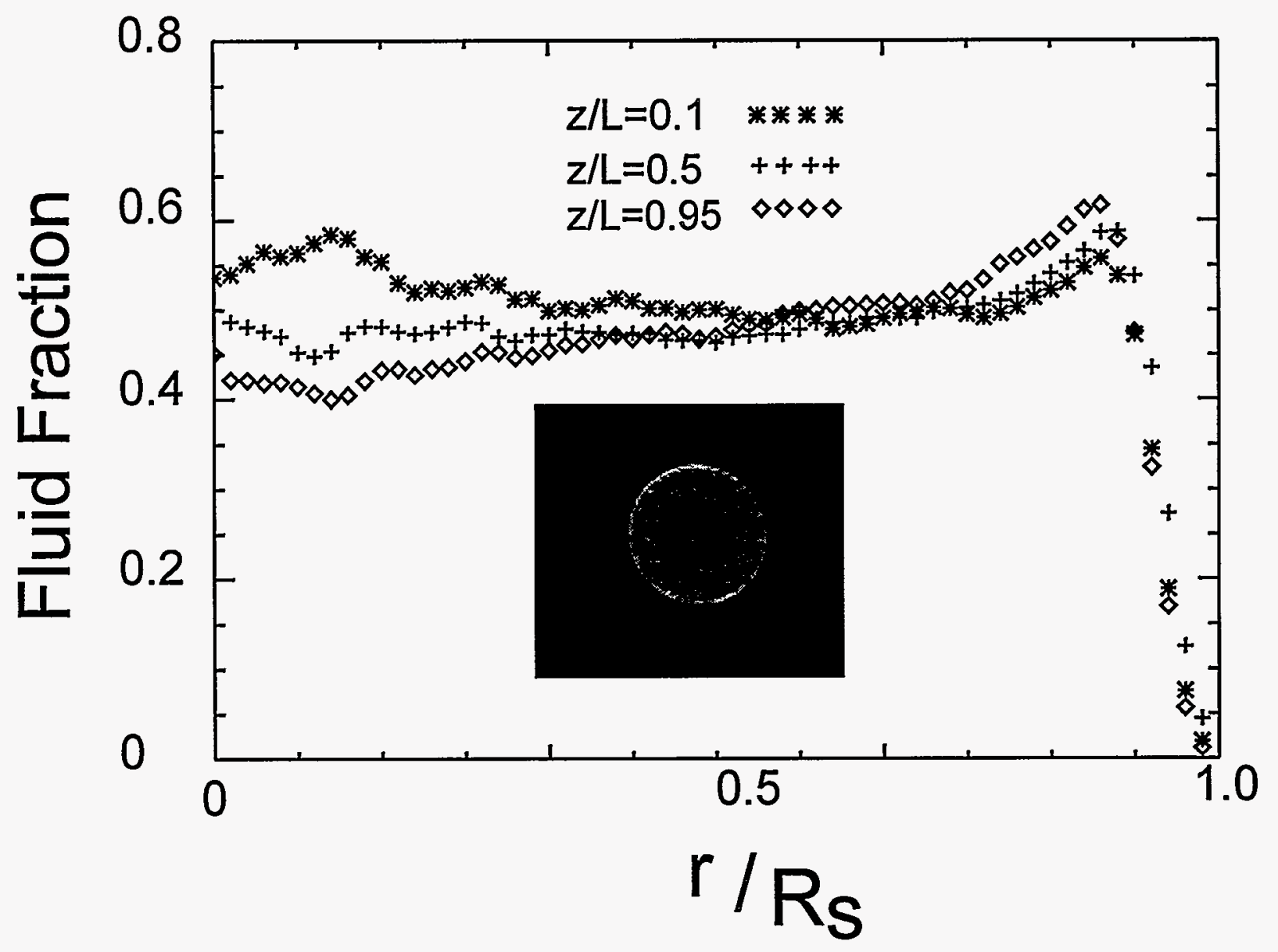

Figure 3 


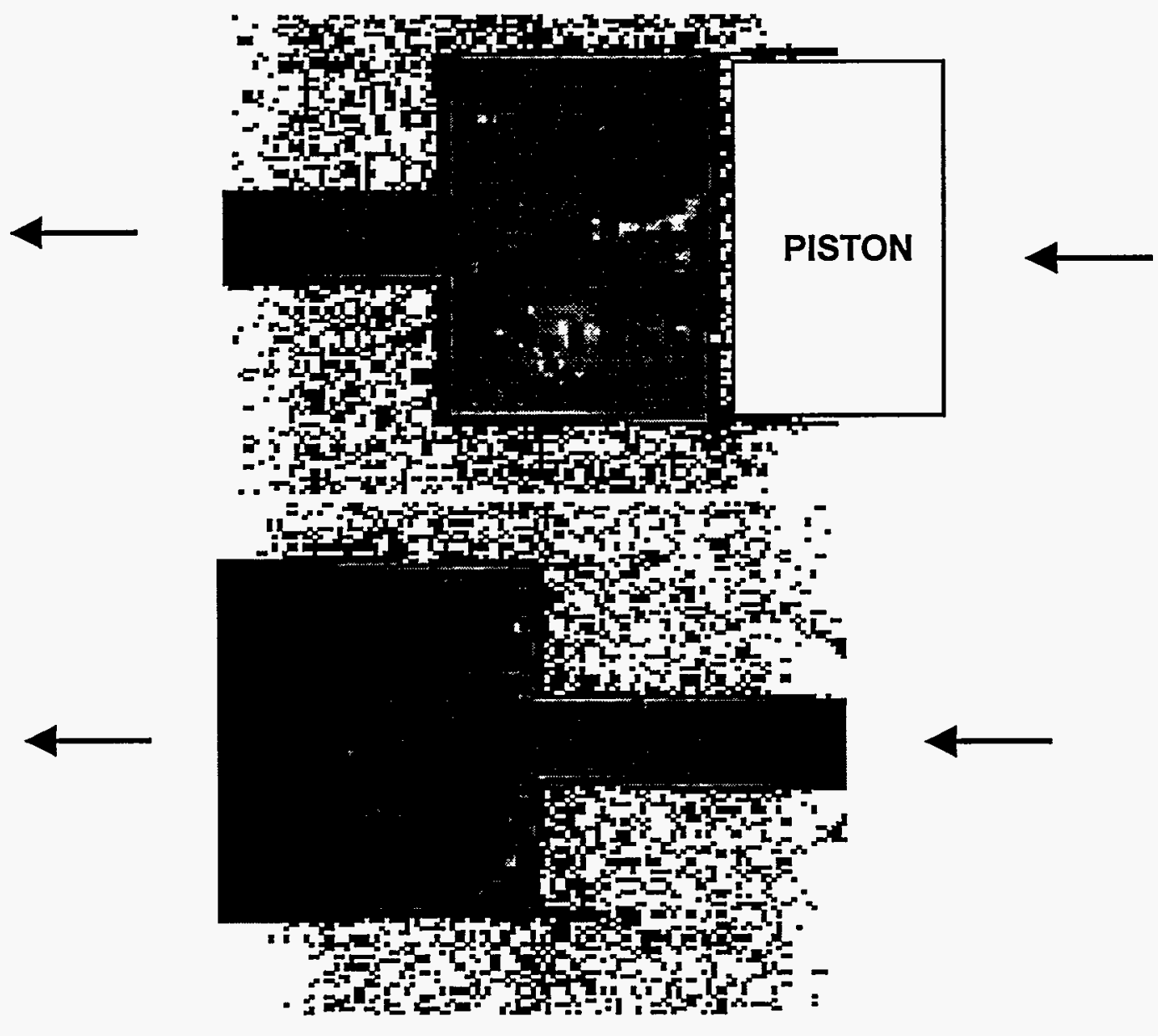

Figure 4 


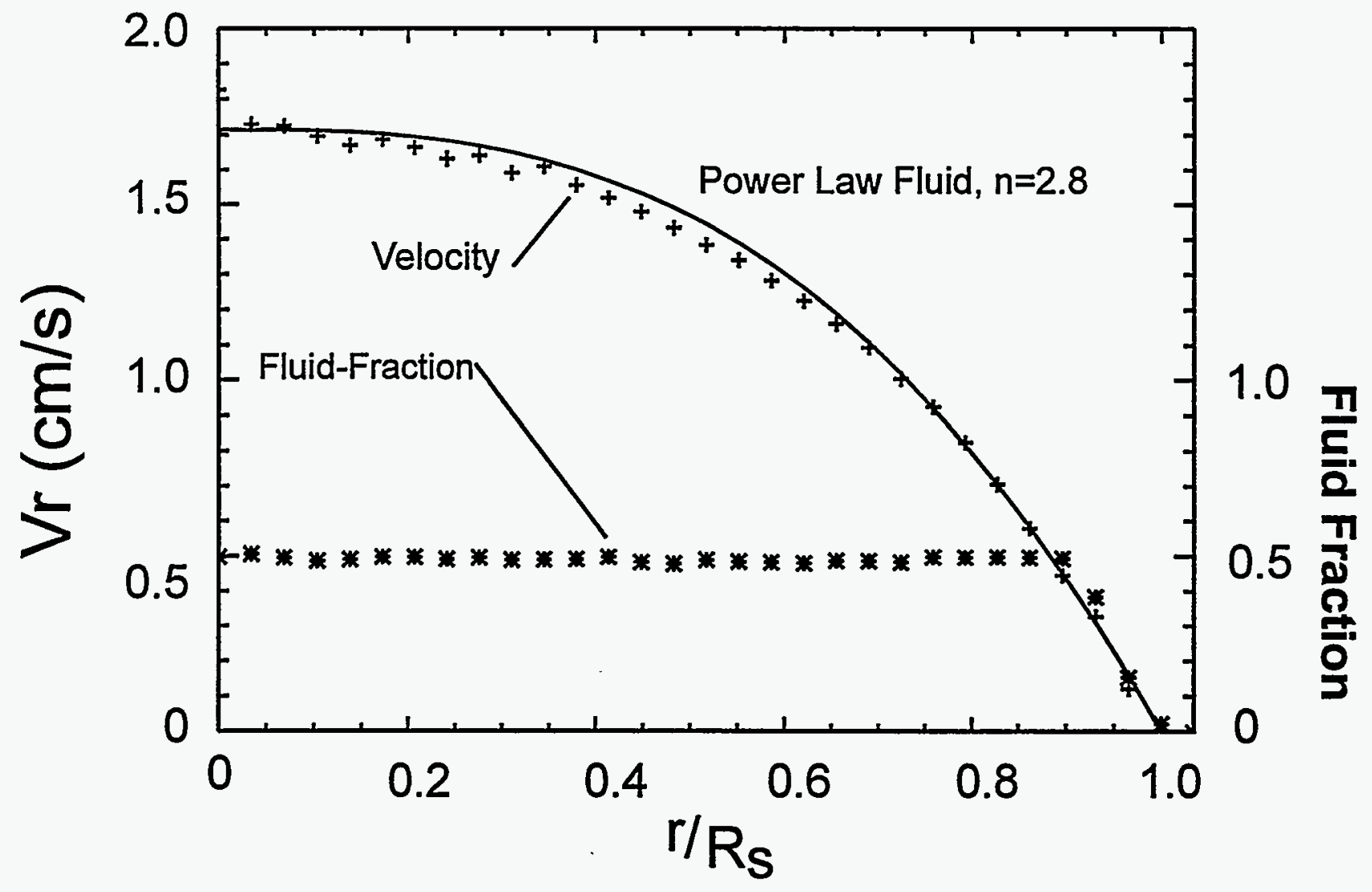

Figure 5 

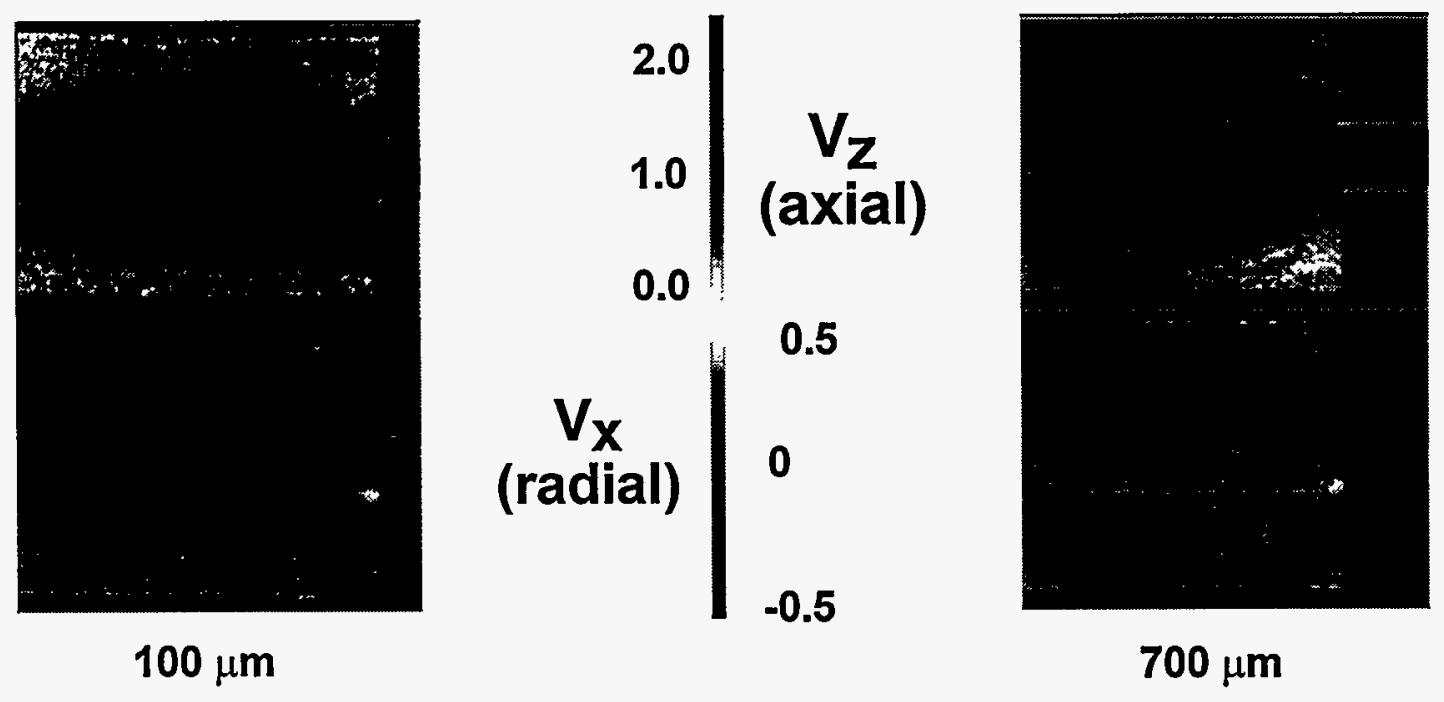

Figure 6 